\title{
q-Fourier Transform and its inversion-problem
}

\author{
M. C. Rocca ${ }^{1,2}$ and A. Plastino ${ }^{1}$ \\ ${ }^{1}$ Instituto de Física (IFLP-CCT-Conicet) \\ Universidad Nacional de La Plata (UNLP) \\ C.C. 67 (1900) La Plata, Argentina \\ 2 Departamento de Física \\ Fac. de Ciencias Exactas, UNLP
}

December 1, 2011

\begin{abstract}
Tsallis' q-Fourier transform is not generally one-to-one. It is shown here that, if we eliminate the requirement that $q$ be fixed, and let it instead "float", a simple extension of the $F_{q}$-definition, this procedure
\end{abstract}


restores the one-to-one character.

KEYWORDS: q-Fourier transform, generalization, ono to one character, statistical mechanics, nonextensive statistical mechanics. 


\section{Introduction}

Nonextensive statistical mechanics (NEXT) 10 2 3, a current generalization of the BoltzmannGibbs (BG) one, is actively studied in diverse areas of Science. NEXT is based on a nonadditive (though extensive 团) entropic information measure characterized by the real index $\mathrm{q}$ (with $\mathrm{q}=1$ recovering the standard BG entropy). It has been applied to variegated systems such as cold atoms in dissipative optical lattices [0, dusty plasmas [6, trapped ions 17, spinglasses [8, turbulence in the heliosheath 9], selforganized criticality [10, high-energy experiments at LHC/CMS/CERN [1] and RHIC/PHENIX/Brookhaven [12, low-dimensional dissipative maps 13, finance 14, galaxies 15, Fokker-Planck equation's applications 16], etc.

NEXT can be advantageously expressed via q-generalizations of standard mathematical concepts (the logarithm and exponential functions, addition and multiplication, Fourier transform (FT) and the Central Limit Theorem (CLT) 17, 22, 25). The q-Fourier transform $F_{q}$ exhibits the nice property of transforming q-Gaussians into q-Gaussians 17. Recently, plane waves, and the representation of the Dirac delta into plane waves have been also generalized [18, 19, 21, 22.

A serious problem afflicts $F_{q}$. It is not generally one-to-one. A detailed 
example is discussed below. In this work we show that by recourse to a rather simple but efficient stratagem that consists in

- eliminating the requirement that $q$ be fixed and instead

- let it "float",

one restores the one-to-one character.

\section{Generalizing the q-Fourier transform}

We define, following [17, a q-Fourier transform of $f(x) \in L^{1}(\mathbb{R}), f(x) \geq 0$ as

$$
\begin{gathered}
F(k, q)=[H(q-1)-H(q-2)] \times \\
\int_{-\infty}^{\infty} f(x)\left\{1+i(1-q) k x[f(x)]^{(q-1)}\right\}^{\frac{1}{1-q}} d x
\end{gathered}
$$

where $H(x)$ is the Heaviside step function.

The only difference between this definition and that given in [17 is that $q$ is not fixed and varies within the interval $[1,2)$. Herein lies the hard-core of our presentation. This simple change of perspective makes it is easy to find the inversion-formula for (2.1) by recourse to the inverse Fourier transform

$$
f(x)=\frac{1}{2 \pi} \int_{-\infty}^{\infty}\left[\lim _{\epsilon \rightarrow 0^{+}} \int_{1}^{2} F(k, q) \delta(q-1-\epsilon) d q\right] e^{-i k x} d k .
$$


As a consequence, we see that this q-Fourier transform is one-to-one, unlike what happens in 23, 24. In the next section we give an illustrative example.

\section{Example}

As an illustration we discuss the example given by Hilhorst in Ref. (22]). Let $f(x)$ be

$$
f(x)=\left\{\begin{array}{l}
\left(\frac{\lambda}{x}\right)^{\beta} ; x \in[a, b] ; 0<a<b ; \lambda>0 \\
0 ; x \text { outside }[\mathrm{a}, \mathrm{b}] .
\end{array}\right.
$$

The corresponding q-Fourier transform is

$$
F(k, q)=\lambda^{\beta} \int_{a}^{b} x^{-\beta}\left\{1+i(1-q) k \lambda^{\beta(q-1)} x^{1-\beta(q-1)}\right\}^{\frac{1}{1-q}} d x .
$$

Effecting the change of variables

$$
y=x^{1-\beta(q-1)},
$$

we have for (3.2)

$$
\begin{gathered}
F(k, q)=[H(q-1)-H(q-2)] \times \\
\frac{\lambda^{\beta}}{1-\beta(q-1)} \int_{a^{1-\beta(q-1)}}^{b^{1-\beta(q-1)}} y^{\frac{\beta(q-2)}{1-\beta(q-1)}}\left\{1+i(1-q) k \lambda^{\beta(q-1)} y\right\}^{\frac{1}{1-q}} d y .
\end{gathered}
$$


Now, (3.3) can be rewritten in the useful form

$$
\begin{gathered}
F(k, q)=[H(q-1)-H(q-2)] \times \\
\left\{\left\{H(q-1)-H\left[q-\left(1+\frac{1}{\beta}\right)\right]\right\} \times\right. \\
\frac{\lambda^{\beta}}{1-\beta(q-1)} \int_{a^{1-\beta(q-1)}}^{b^{1-\beta(q-1)}} y^{-\frac{\beta(2-q)}{1-\beta(q-1)}}\left\{1+i(1-q) k \lambda^{\beta(q-1)} y\right\}^{\frac{1}{1-q}} d y+ \\
\left\{H\left[q-\left(1+\frac{1}{\beta}\right)\right]-H(q-2)\right\} \times \\
\left.\frac{\lambda^{\beta}}{\beta(q-1)-1} \int_{a^{1-\beta(q-1)}} y^{\frac{\beta(q-2)}{1-\beta(q-1)}}\left\{1+i(1-q) k \lambda^{\beta(q-1)} y\right\}^{\frac{1}{1-q}} d y\right\} .
\end{gathered}
$$

Taking into account that the involved integrals are defined in a finite interval,

we can cast (3.4) as

$$
\begin{gathered}
F(k, q)=[H(q-1)-H(q-2)] \times \\
\left\{\left\{H(q-1)-H\left[q-\left(1+\frac{1}{\beta}\right)\right]\right\} \times\right. \\
\frac{\lambda^{\beta}}{1-\beta(q-1)} \lim _{\epsilon \rightarrow 0^{+}} \int_{a^{1-\beta(q-1)}}^{b^{1-\beta(q-1)}} y^{-\frac{\beta(2-q)}{1-\beta(q-1)}}\left\{1+i(1-q)(k+i \epsilon) \lambda^{\beta(q-1)} y\right\}^{\frac{1}{1-q}} d y+ \\
\left\{H\left[q-\left(1+\frac{1}{\beta}\right)\right]-H(q-2)\right\} \times \\
\left.\frac{\lambda^{\beta}}{\beta(q-1)-1} \lim _{\epsilon \rightarrow 0^{+}} \int_{b^{1-\beta(q-1)}} y^{\frac{\beta(q-2)}{1-\beta(q-1)}}\left\{1+i(1-q)(k+i \epsilon) \lambda^{\beta(q-1)} y\right\}^{\frac{1}{1-q}} d y\right\} .
\end{gathered}
$$


We now use results of the Integral's table 26 to evaluate (3.5) and get

$$
\begin{gathered}
\lim _{\epsilon \rightarrow 0^{+}} \int_{a^{1-\beta(q-1)}}^{\infty} y^{-\frac{\beta(2-q)}{1-\beta(q-1)}}\left\{1+i(1-q)(k+i \epsilon) \lambda^{\beta(q-1)} y\right\}^{\frac{1}{1-q}} d y= \\
\frac{(q-1)[1-\beta(q-1)] a^{\frac{q-2}{q-1}}}{(2-q)\left[(1-q) i(k+i 0) \lambda^{\beta}\right]^{\frac{1}{q-1}}} \times \\
F\left(\frac{1}{q-1}, \frac{2-q}{(q-1)[1-\beta(q-1)]}, \frac{1}{q-1}+\frac{\beta(2-q)}{1-\beta(q-1)}\right. \\
\left.-\frac{1}{(1-q) i(k+i 0) \lambda^{\beta(q-1)} a^{1-\beta(q-1)}}\right)
\end{gathered}
$$

and

$$
\begin{gathered}
\lim _{\epsilon \rightarrow 0^{+}} \int_{0}^{a^{1-\beta(q-1)}} y^{\frac{\beta(2-q)}{\beta(q-1)-1}}\left\{1+i(1-q)(k+i \epsilon) \lambda^{\beta(q-1)} y\right\}^{\frac{1}{1-q}} d y= \\
\frac{[\beta(q-1)-1] a^{1-\beta}}{\beta-1} \times \\
F\left(\frac{1}{q-1}, \frac{\beta-1}{\beta(q-1)-1}, \frac{\beta q-2}{\beta(q-1)-1} ;\right. \\
\left.(q-1) i(k+i 0) \lambda^{\beta(q-1)} a^{1-\beta(q-1)}\right),
\end{gathered}
$$

where $F(a, b, c ; z)$ is the hypergeometric function. Thus we obtain for $F(k, q)$

$$
\begin{gathered}
F(k, q)=[H(q-1)-H(q-2)] \times \\
\left\{\left\{H(q-1)-H\left[q-\left(1+\frac{1}{\beta}\right)\right]\right\} \times\right. \\
\frac{(q-1) \lambda^{\beta}}{(2-q)\left[(1-q) i(k+i 0) \lambda^{\beta}\right]^{\frac{1}{q-1}}} \times \\
\left\{a ^ { \frac { q - 2 } { q - 1 } } F \left(\frac{1}{q-1}, \frac{2-q}{(q-1)[1-\beta(q-1)]}, \frac{1}{q-1}+\frac{\beta(2-q)}{1-\beta(q-1)} ;\right.\right.
\end{gathered}
$$




$$
\begin{gathered}
\left.\frac{1}{(q-1) i(k+i 0) \lambda^{\beta(q-1)} a^{1-\beta(q-1)}}\right)- \\
b^{\frac{q-2}{q-1}} F\left(\frac{1}{q-1}, \frac{2-q}{(q-1)[1-\beta(q-1)]}, \frac{1}{q-1}+\frac{\beta(2-q)}{1-\beta(q-1)}\right. \\
\left.\left.\quad \frac{1}{(q-1) i(k+i 0) \lambda^{\beta(q-1)} b^{1-\beta(q-1)}}\right)\right\}+ \\
\left\{H\left[q-\left(1+\frac{1}{\beta}\right)\right]-H(q-2)\right\} \frac{\lambda^{\beta}}{\beta-1} \times \\
\left\{a ^ { 1 - \beta } F \left(\frac{1}{q-1}, \frac{\beta-1}{\beta(q-1)-1}, \frac{\beta q-2}{\beta(q-1)-1}\right.\right. \\
\left.(q-1) i(k+i 0) \lambda^{\beta(q-1)} a^{1-\beta(q-1)}\right)- \\
b^{1-\beta} F\left(\frac{1}{q-1}, \frac{\beta-1}{\beta(q-1)-1}, \frac{\beta q-2}{\beta(q-1)-1}\right. \\
\left.\left.\left.(q-1) i(k+i 0) \lambda^{\beta(q-1)} b^{1-\beta(q-1)}\right)\right\}\right\} .
\end{gathered}
$$

As we can see from (3.8), $F(k, q)$ is dependent of $a$ and $b$, and, as consequence, one-to-one as has been shown in Section 2 .

However, and this is the crucial issue, if we fix $q$ and select $\beta=1 /(q-1)$ (3.8) simplifies and adopts the appearance

$$
\begin{gathered}
F(k, q)=\lambda^{\frac{1}{q-1}} \frac{q-1}{2-q}[H(q-1)-H(q-2)] \times \\
{\left[a^{\frac{q-2}{q-1}} F\left(\frac{1}{q-1}, \frac{2-q}{q-1}, \frac{2-q}{q-1} ;(q-1) i(k+i 0) \lambda\right)-\right.} \\
\left.b^{\frac{q-2}{q-1}} F\left(\frac{1}{q-1}, \frac{2-q}{q-1}, \frac{2-q}{q-1} ;(q-1) i(k+i 0) \lambda\right)\right] .
\end{gathered}
$$


With the help of the result given in 27 for

$$
F(-a, b, b,-z)=(1+z)^{a}
$$

we obtain for (3.9):

$$
\begin{gathered}
F(k, q)=\lambda^{\frac{1}{q-1}} \frac{q-1}{2-q}[H(q-1)-H(q-2)] \\
\left(a^{\frac{q-2}{q-1}}-b^{\frac{q-2}{q-1}}\right)[1+(1-q) i k \lambda]^{\frac{1}{1-q}} .
\end{gathered}
$$

Using now the expression for $\lambda$ of 22, i.e.,

$$
\lambda=\left[\left(\frac{q-1}{2-q}\right)\left(a^{\frac{q-2}{q-1}}-b^{\frac{q-2}{q-1}}\right)\right]^{1-q},
$$

we have, finally,

$$
F(k, q)=[H(q-1)-H(q-2)][1+(1-q) i k \lambda]^{\frac{1}{1-q}}
$$

which is the result given by Hilthorst in 22, demonstrating that $F(k, q)$ is not one-to-one. As a conclusion we can say that for fixed $q$ the q-Fourier transform is NOT one-to-one. On the contrary, as we have shown in section 2, when q is NOT fixed, the q-Fourier transform is indeed one-to-one.

\section{Conclusions}

In the present communication we have discussed the NOT one-to-one nature of the q-Fourier transform $F_{q}$. We have shown that, if we eliminate the 
requirement that $q$ be fixed and let it "float" instead, such simple extension of the $F_{q}$-definition restores the desired one-to-one character.

Acknowledments The authors thank Prof. C. Tsallis for having called our attention to the present problem. 


\section{References}

[1] C. Tsallis, J. Stat. Phys. 52 (1988) 479.

[2] M. Gell-Mann, C. Tsallis (Eds.), Nonextensive Entropy Interdisciplinary Applications, Oxford University Press, New York, 2004; C. Tsallis, Introduction to Nonextensive Statistical Mechanics Approaching a Complex World, Springer, New York, 2009.

[3] A. R. Plastino, A. Plastino, Phys. Lett A 177 (1993) 177.

[4] C. Tsallis, M. Gell-Mann, Y. Sato, Proc. Natl. Acad. Sci. USA 102 (2005) 15377; F. Caruso, C. Tsallis, Phys. Rev. E 78 (2008) 021102.

[5] P. Douglas, S. Bergamini, F. Renzoni, Phys. Rev. Lett. 96 (2006) 110601; G.B. Bagci, U. Tirnakli, Chaos 19 (2009) 033113.

[6] B. Liu, J. Goree, Phys. Rev. Lett. 100 (2008) 055003.

[7] R.G. DeVoe, Phys. Rev. Lett. 102 (2009) 063001.

[8] R.M. Pickup, R. Cywinski, C. Pappas, B. Farago, P. Fouquet, Phys. Rev. Lett. 102 (2009) 097202.

[9] L.F. Burlaga, N.F. Ness, Astrophys. J. 703 (2009) 311. 
[10] F. Caruso, A. Pluchino, V. Latora, S. Vinciguerra, A. Rapisarda, Phys. Rev. E 75 (2007) 055101(R); B. Bakar, U. Tirnakli, Phys. Rev. E 79 (2009) 040103(R); A. Celikoglu, U. Tirnakli, S.M.D. Queiros, Phys. Rev. E $82(2010) 021124$.

[11] V. Khachatryan, et al., CMS Collaboration, J. High Energy Phys. 1002 (2010) 041; V. Khachatryan, et al., CMS Collaboration, Phys. Rev. Lett. 105 (2010) 022002.

[12] Adare, et al., PHENIX Collaboration, Phys. Rev. D 83 (2011) 052004; M. Shao, L. Yi, Z.B. Tang, H.F. Chen, C. Li, Z.B. Xu, J. Phys. G 37 (8) (2010) 085104.

[13] M.L. Lyra, C. Tsallis, Phys. Rev. Lett. 80 (1998) 53; E.P. Borges, C. Tsallis, G.F.J. Ananos, P.M.C. de Oliveira, Phys. Rev. Lett. 89 (2002) 254103; G.F.J. Ananos, C. Tsallis, Phys. Rev. Lett. 93 (2004) 020601; U. Tirnakli, C. Beck, C. Tsallis, Phys. Rev. E 75 (2007) 040106(R); U. Tirnakli, C. Tsallis, C. Beck, Phys. Rev. E 79 (2009) 056209.

[14] L. Borland, Phys. Rev. Lett. 89 (2002) 098701.

[15] A. R. Plastino, A. Plastino, Phys. Lett A 174 (1993) 834. 
[16] A. R. Plastino, A. Plastino, Physica A 222 (1995) 347.

[17] S. Umarov, C. Tsallis, S. Steinberg, Milan J. Math. 76 (2008) 307; S. Umarov, C. Tsallis, M. Gell-Mann, S. Steinberg, J. Math. Phys. 51 (2010) 033502.

[18] M. Jauregui, C. Tsallis, J. Math. Phys. 51 (2010) 063304.

[19] A. Chevreuil, A. Plastino, C. Vignat, J. Math. Phys. 51 (2010) 093502.

[20] M. Mamode, J. Math. Phys. 51 (2010) 123509.

[21] A. Plastino and M.C.Rocca: J. Math. Phys 52, 103503 (2011).

[22] H.J.Hilhorst: J. Stat. Mech. P10023 (2010)

[23] M.Jauregui and C.Tsallis: Phys. Lett. A 375, 2085 (2011).

[24] M.Jauregui, C.Tsallis and E.M.F. Curado: arXiv:1108.2690v1.

[25] M. Jauregui, C, Tsallis, Phys. Lett. A 375 (2011) 2085.

[26] L. S. Gradshtein and I. M. Ryzhik : Table of Integrals, Series, and Products. Fourth edition, Academic Press (1965) 3.1941 and 3.1942 pages 284 and 285 . 
[27] M.Abramowitz and I.A.Stegun: Handbook of Mathematical Functions. National Bureau of Standards. Applied Mathematical Series 55 Tenth Printing (1972), 15.1 .8 page 556.

[28] L. S. Gradshtein and I. M. Ryzhik : Table of Integrals, Series, and Products. Fourth edition, Academic Press (1965) 3.1943 page 285. 